\title{
EXTRACTION OF FREQUENCY DEPENDENT MACRO-MODELS FOR GAS DAMPING AND SPRING EFFECTS FOR MEMS DEVICES
}

\author{
Yao-Joe Yang, Mattan Kamon, Vladimir L. Rabinovich, Chahid Ghaddar, \\ Manish Deshpande, Ken Greiner, and John R. Gilbert
}

Microcosm Technologies Inc.,
Cambridge, MA 02142, USA

\begin{abstract}
In this paper, we present an efficient macromodel extraction technique for gas damping and spring effects for arbitrarily shaped geometry. The technique applies an Arnoldi-based model-order-reduction algorithm to generate low-order models from a FEM approximation of the linearized Reynolds equation. We demonstrate that this approach for generating the frequency-dependent gas-damping model is more than 100 times faster than previous approaches, which solves the linearized Reynolds equation using a transient finite-element method. The low-order gas-damping model can be easily inserted into a system-level modeling package for transient and frequency analysis. The simulated results arc in good agreement with experimental results for four different devices.
\end{abstract}

\section{INTRODUCTION}

A large class of MEMS devices must operate at significant gas pressures. To understand the dynamic behavior of these devices the effects of the gas surrounding the movable component can be critical. Devices such as accelerometers, gyroscopes, switches, optical modulators, micromirrors, and resonant sensors all require an understanding of gas damping and spring effects for accurate modeling.

Most work in damping has focused on getting more accurate simulations of the small or large signal 3D gas damping and spring effects [1-7]. The typical results of such analysis are similar to Figure 1, which shows the gas damping and spring components vs. frequency for the microrelay [8] shown in Figure 2(a). This paper is about the development of efficient techniques to take the solid models of full 3D structures and automatically cxtract spice-like time domain system models to accurately represent the frequency dependent damping and spring forces.

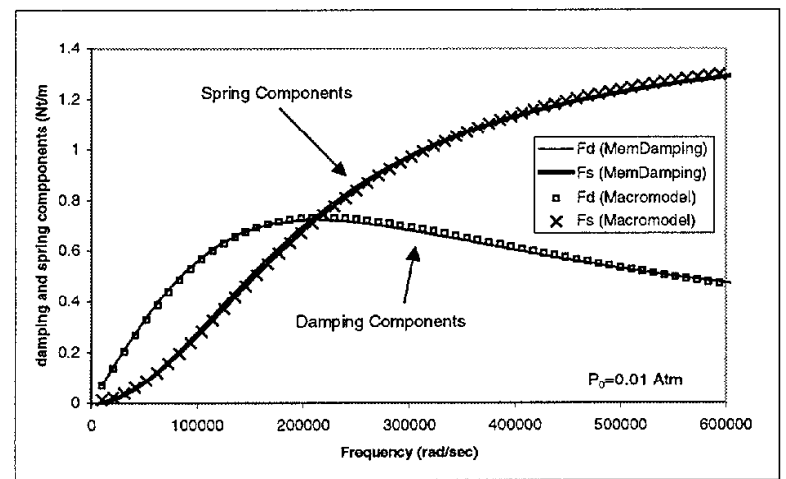

Figure 1 Results of $3 D$ physics analysis of gas spring and damping for beam switch of Figure 2(a), operating in its first vibration mode. The solid lines are obtained using MemDamping [9]. The points show the overlap of the macromodel obtained from the Arnoldi-based model-orderreduction technique.
The extraction procedure is shown in Figure 3. A 3D model of a MEMS device is built using MEMCAD [9]. The finiteelement (FE) matrix system for solving transient small signal analysis such as NSR or linearized Reynolds is constructed and then reduced to a low order system using the Arnoldi-based PRIMA algorithm [10]. After the reduced system is scaled with proper gap-dependent coefficients, it is then converted to a time domain representation written in an HDL, in this case MAST for use in SABER.

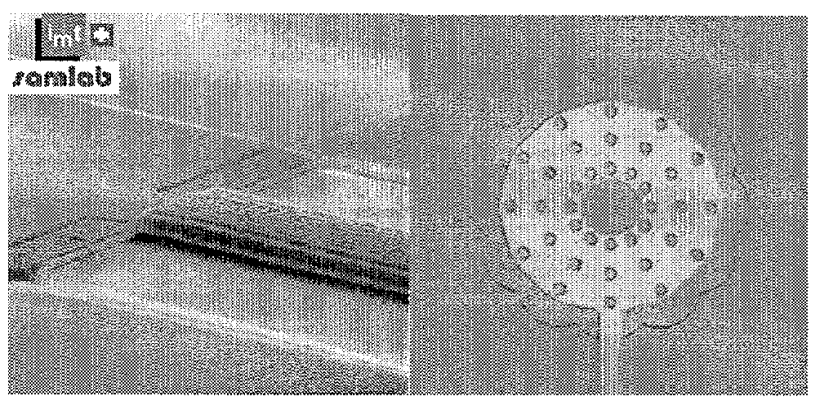

(a)

(b)

Figure 2 (a) SEM picture of a microrelay from IMT. (b) An optical micrograph of a high frequency optical modulator from Lucent Technology.

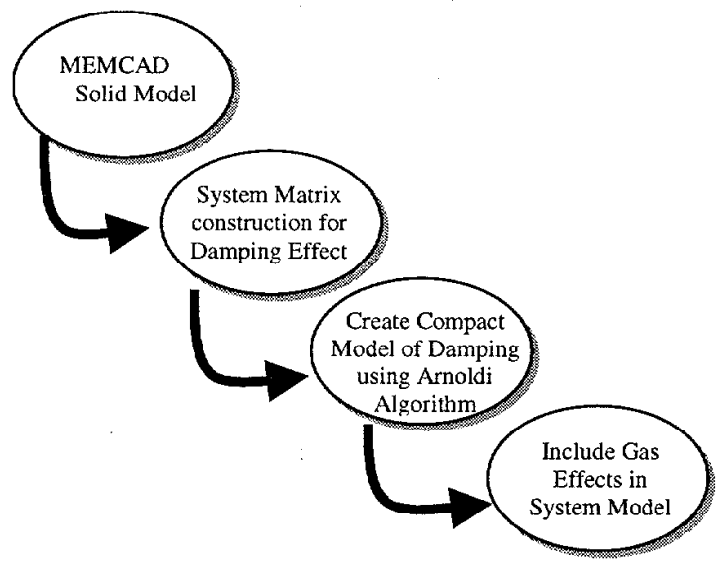

Figure 3 Procedure of the efficient and accurate air damping modeling described in this paper

Note that this new approach does not require the meshing of air gaps that contribute to gas-damping effects. The effect of perforations and the effect of viscous damping on plate edges [7] are also incorporated into the FE matrix system in the model construction step. Because the model is generated directly from the FE system, the transient calculations of the full FEM model for a wide frequency range, as reported in [5], is not performed. This brings the total computation time to obtain a frequency- 
dependent gas damping model to a few minutes vs. a few hours/days in previous approaches $[3,5,7]$.

This work complements prior work in macro-model cxtraction for electromechanics that has succeeded in extracting $6 \mathrm{DOF}$ models for a general electromechanical plate on tether systems [11]. We can now extract full systems such as the electromechanical switch schematic of Figure 4.

\section{THEORY}

The models are extracted using the Arnoldi-based model reduction algorithm known as PRIMA [10], which is commonly used for model reduction of electrical interconnects. Our approach is similar to the model reduction approach used in [12]. In [12], model reduction was applied to a linearized form of the fully coupled electro-mechanical-fluid damped system. Here, we treat the squeeze film damping separately and build modcls in the mechanical mode shape basis. Such an approach would allow these models to be readily combined with the mode shape based models in the low order model of the entire system [13]. Additionally, we show that the models can be used for large-signal motion with certain restrictions.

To begin, the linearized Reynolds equation for squeeze film damping from [1] is

$$
\frac{h_{0}}{P_{0}} \cdot \frac{\partial p}{\partial t}=\frac{h_{0}^{3}}{12 \mu} \nabla^{2} p-\frac{\partial e}{\partial t}
$$

where the variation in plate spacing $h$ is assumed to be small compared to the mean spacing, $h_{0}$, given by

$$
h=h_{0}+e(x, t)
$$

with $x \in \Re^{2}$ and $e \ll h_{0}$. The variation in pressure, $P$, will thus be small compared to ambient,

$$
P=P_{0}+p(x, t)
$$

Equation (1) can be solved by finite element analysis for a given $e(x, t)$. Let $f(x)$ be the shape (perhaps mode shape) of the displacement so that $e(x, t)=f(x) \cdot u(t)$. The dynamic system from discretizing (1) by finite elements can be written in state space form as

$$
\begin{aligned}
& \frac{h_{0}}{P_{0}} B \frac{d \mathbf{p}}{d t}=\frac{h_{0}^{3}}{12 \mu} A \mathbf{p}+B \mathbf{f} u(t) \\
& y=(B \mathbf{f})^{T} \mathbf{p}
\end{aligned}
$$

where $A, B \in \Re^{n \times n}$, where $\mathrm{n}$ is the number of nodal degrees of freedom, $\mathbf{p}$ is the pressure at the nodes, and $\mathbf{f}$ is $f(x)$ evaluated at the node points. $y$ is then the net force projected into the shape defined by $f(x)$ in a finite element sense.

The dynamic system of (2) is too large to insert directly into a system simulator such as SPICE or SABER. We thus apply PRIMA to generate a low order representation of (2) which still accurately captures the dynamic behavior. To apply the PRIMA algorithm to generate a $\mathrm{k}$-th order model, $\mathrm{k}$ orthogonal vectors $\left\{v_{i}\right\} \in \Re^{n}$ are computed which span the vector space known as Krylov subspace:

$$
K_{k}=\left\{(B \mathbf{f}), A^{1} B(B \mathbf{f}), \ldots,\left(A^{1} B\right)^{k-1}(B \mathbf{f})\right\}
$$

These vectors can be stably computed via the Arnoldi algorithm [10]. Given the matrix $V$ whose columns are $\left\{v_{i}\right\}$, the reduced order model is

$$
\begin{aligned}
& \frac{h_{0}}{P_{0}} \widetilde{B} \frac{d \tilde{\mathbf{p}}}{d t}=\frac{h_{0}^{3}}{12 \mu} \tilde{A} \tilde{\mathbf{p}}+\tilde{\mathbf{f}} u(t) \\
& y=\tilde{\mathbf{f}}^{T} \tilde{\mathbf{p}}
\end{aligned}
$$

where $\tilde{B}=V^{T} B V, \tilde{A}=V^{T} A V$, and $\tilde{\mathbf{f}}=V^{T} B \mathbf{f}$. The attractive properties of such an approach are that the first $\mathrm{k}$ Taylor series coefficients of the transfer function of (3) match those of the original model in (1). In addition, the model is guaranteed to be passive. Finally, the method easily extends to a single model with multiple inputs $\left\{u_{1}(x), u_{2}(x), \ldots.\right\}$ corresponding to multiple mode shapes, $\left\{\mathbf{f}_{1}(x), \mathbf{f}_{2}(x), \ldots\right\}$.

As will be seen in the next section, $\mathrm{k}=5$ is generally adequate for an accurate damping model. This low order model can be inserted directly into a system simulator such as SPICE or SABER. Note that since the vector space spanned by $\left\{v_{i}\right\}$ does not depend on the mean gap, ambient pressure or viscosity, the above model is valid for any choice of those parameters. Going one step further, we can model large signal behavior by letting the mean gap vary with time, $h_{0}=h_{0}(t)$. Such an approach would be valid if $h_{0}(t)$ varies slowly compared to $u(t)$. In fact, from numerical experiment, we find that replacing $h_{0}$ in (2) with $h_{0}(t)=h_{0}+u(t)$ for even large $u(t)$ gives good results.

\section{COMPARISON TO EXPERIMENTAL RESULTS}

Once we obtain a reduced-order model (macromodel) for gas damping from the PRIMA algorithm, an HDL template of the gas model written in MAST is automatically generated. A Saber system-level model of a whole MEMS device with this frequency-dependant gas-damping macromodel can be easily built using Saber, and small signal as well as transient analyses can he simulated. Figure 4 is an example of a Saber system-level model of a microswitch [14], which includes a lumped inertia, two mechanical springs, two automatically-generated frequencydependent gas-damping models for two gaps, a electrostatic force actuator with a voltage source, and two mechanical stoppcrs.

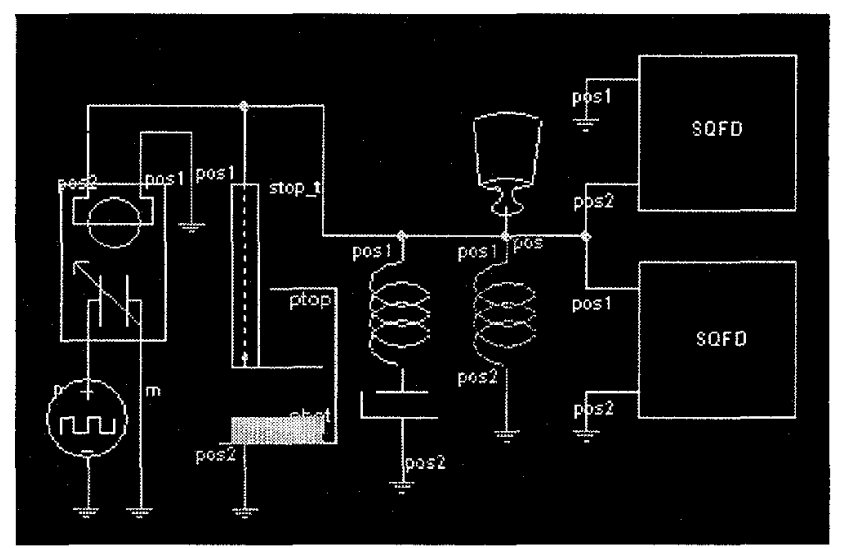

Figure 4 Schernatic of a full MEMS switch [13] model including components for an inertia, two mechanical springs, an electrostatic actuator, a voltage source, two mechanical stoppers and two extracted macromodels for gas induced forces. 


\section{A. Small signal oscillation with or without mode shapes}

Three experimental data sets are used to verify the gasdamping models generated by the Arnoldi-based algorithm: a microrelay from IMT [8], optical modulators from Lucent Technology [15] (shown in Figure 2(b)), and a low-frequency accelerometer from Motorola (shown in Figure 5). Figure 6 shows the frequency response (small $\mathrm{AC}$ analysis by Saber) of an IMT microrelay for different ambient pressures. The fixedfixed-beam microrelay operates in its first oscillation mode. The changes in quality factors as well as resonance shifting effects can be easily observed in the figure as pressure varies.

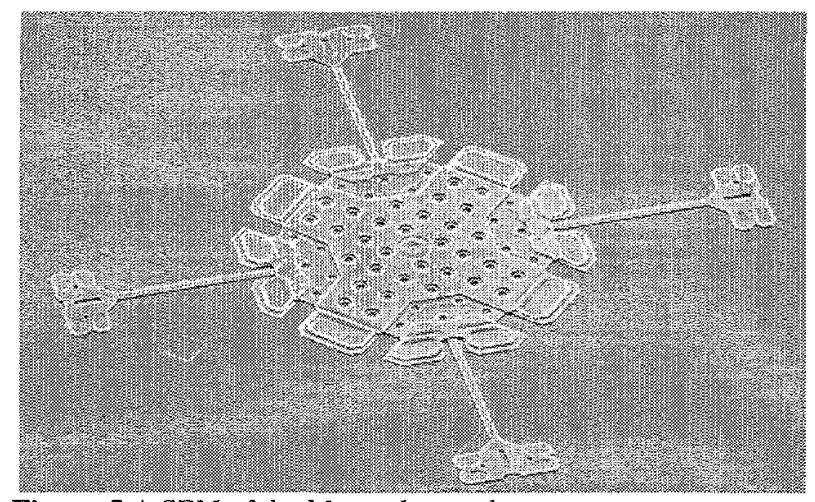

Figure 5 A SEM of the Motorola accelerometer

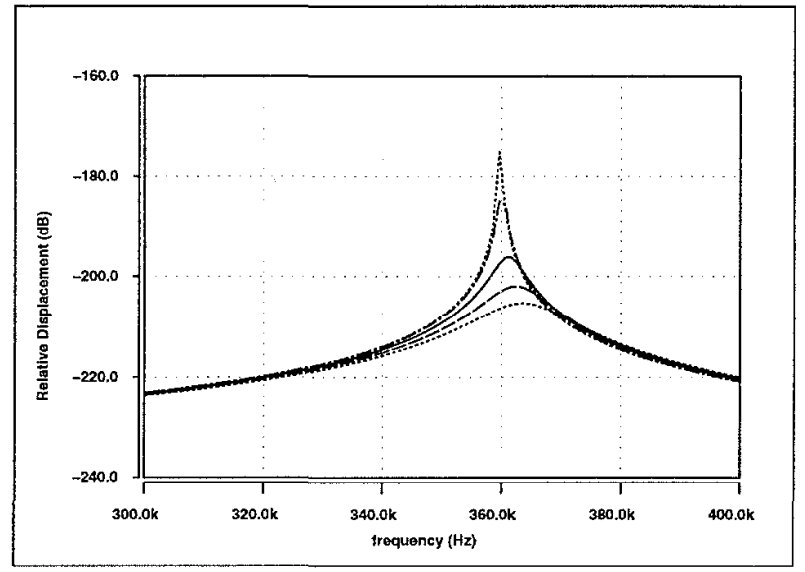

Figure 6 Frequency response of an IMT microrelay for different pressures of $0.1,1,5,10$ and 15 mbar (curves from top to bottom). The quality factors and resonance shifting effects can be easily extracted from the curves.

Figure 7 shows the gas damping coefficients vs. frequency for these three devices. The gas spring constant vs. frequency is shown in Figure 8. The simulated results in these figures are generated by reduced models of order 5 . Note that the experimental and simulated results are in good agreement spanning about 6 orders of magnitude in frequency.

The comparison of computational times for calculating the frequency-dependent damping the spring components (e.g., Figure 1) is shown in Table 1. In the previous approaches $[3,5,7]$, at least 20 transient simulations with different smallamplitude-oscillation frequencies were needed to generate the damping and spring components spanning across a desired frequency range. The new approach is a least 100 times faster than the previous approaches. It also automatically builds a time-domain model for system-level analysis.

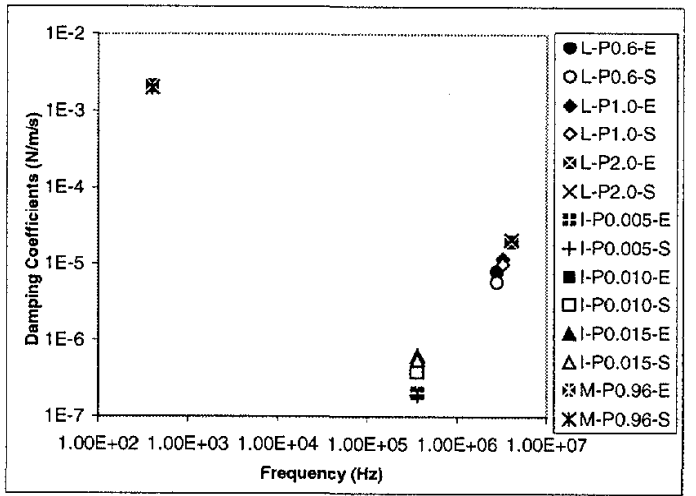

Figure 7 Gas damping coefficients vs. Frequency for three devices. (L: Lucent, M: Motorola, I: IMT; P\#.\#: pressure at \#.\# bar; $E$ : experiment data, $S$ : simulation data)

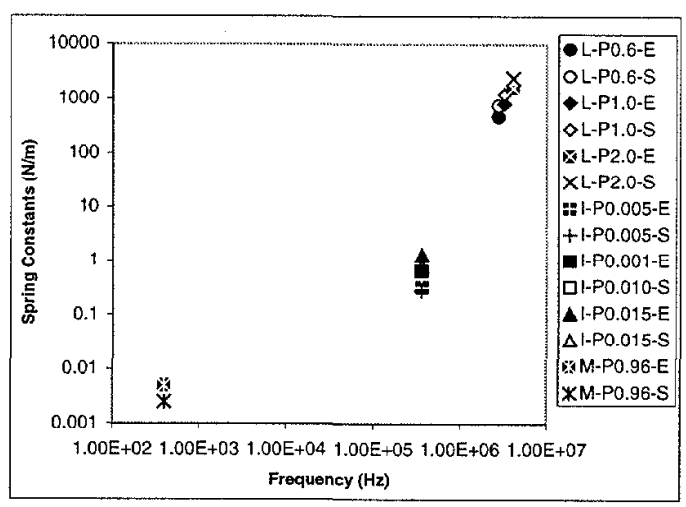

Figure 8 Gas spring constants vs. Frequency for three devices. (L: Lucent, M: Motorola, I: IMT; P\#.\#: pressure at \#.\# bar; E: experiment data, S: simulation data)

\begin{tabular}{|c|c|c|}
\cline { 2 - 3 } \multicolumn{1}{c|}{} & \multicolumn{2}{c|}{ Total Computation Times } \\
\cline { 2 - 3 } \multicolumn{1}{c|}{} & Previous Approach & New Approach \\
\hline Lucent & $948 \mathrm{~min}$ & $1.5 \mathrm{~min}$ \\
\hline Motorola & $431 \mathrm{~min}$ & $1.1 \mathrm{~min}$ \\
\hline IMT & $200 \mathrm{~min}$ & $0.4 \mathrm{~min}$ \\
\hline
\end{tabular}

Table 1 Comparison of computation times between previous and new approaches

\section{B. Transient Analysis}

Figure 9 shows experimental and simulated transient responses of a microswitch which schematic is shown in Figure 4. A step voltage applied between the plate and substrate for 1 ms. The plate collapses on the stopper, then is released from the substrate (after turning off the applied voltage) and oscillates around its neutral position.

The large amplitude macromodel transient results are also compared with the full non-linear Reynolds simulations [3] using finite-difference method (FDM). Figure 10 and Figure 11 show the transient results for a square plate on tethers over a counter electrode with applying step voltages of 2 Volts (below pull-in vollage) and 3 Volts (above pull-in voltage). The ambient pressure is $100 \mathrm{~N} / \mathrm{m}^{2}$, the gap is $2 \mu \mathrm{m}$, and the plate is $500 \times 500$ $\mu \mathrm{m}^{2}$. Below pull-in voltage, the macromodel and the FDM results match very well, as shown in Figure 10 . However, the results of the macromodel with fixed $h_{0}$ (see equation 2) underestimates the damping as the plate moves away from its original gap. 
When applied voltage is larger than pull-in voltage, the plate velocity increases significantly when plate is close to touch down, which in turn significantly increases the pressure back force against the plate. This large pressure increase breaks the linearized Reynolds equation assumption that the pressure variation is much less than ambient pressure. The macromodel result diverges from the FDM result after the plate travels across half gap, as shown in Figure 11, due to this highly nonlinear pressure force. The result of the macromodel with fixed $h_{o}$ starts to diverge from the FDM result after the plate travels about a quarter of the gap.

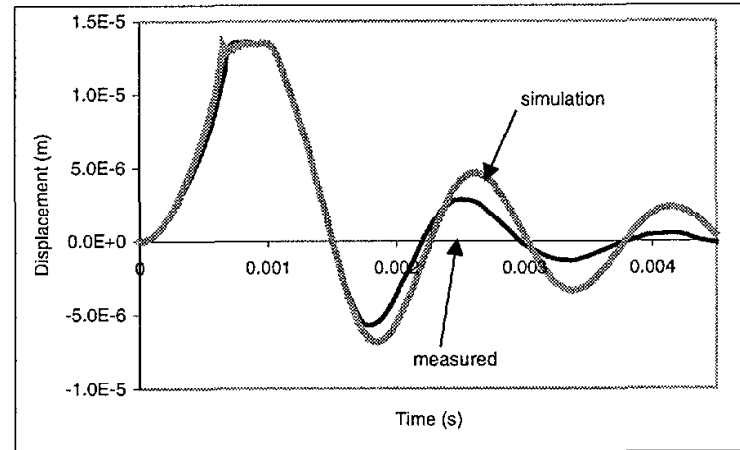

Figure 9 Comparison between Experimental transient in MEMS switch and simulation using extracted macromodels for gas damping and spring effects. Experimental data is from Minami, et. al [14].

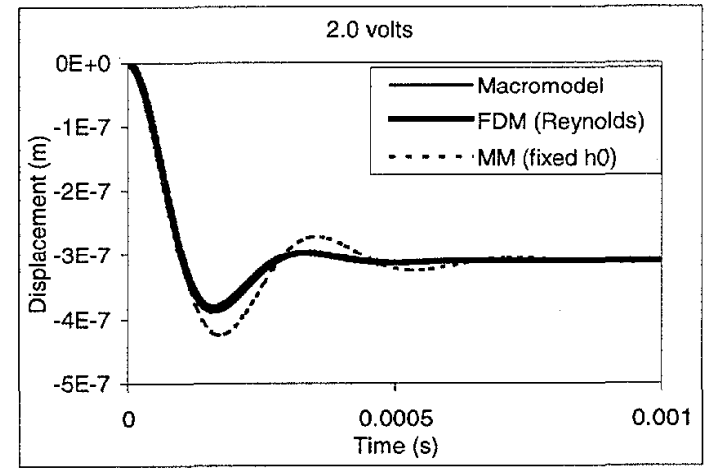

Figure $10 \mathrm{~A}$ comparison among macromodel, macromodel with fixed $h_{0}$, and full non-linear Reynolds for large displacement transient behavior. The applying voltage is $2 \mathrm{~V}$.

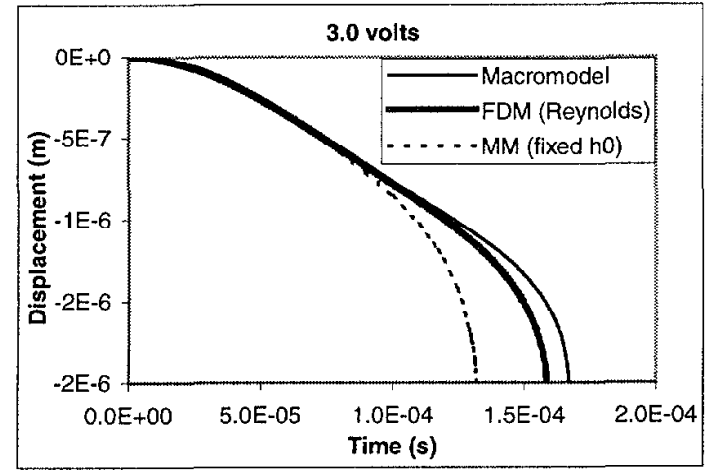

Figure $11 \mathrm{~A}$ comparison among macromodel, macromodel with fixed $h_{0}$, and full non-linear Reynolds for pull-in transient behavior. The applying voltage is $3 \mathrm{~V}$.

\section{CONCLUSION}

A new approach to extract frequency-dependent gas damping models for arbitrary geometries is demonstrated. The Arnoldi-based algorithm is applied for creating a low-order model from the transient FE system matrices of NSR analysis. The frequency-dependant gas damping and spring effects can be obtained using the low-order models without any computationally intensive transient simulation for wide frequency range. After constructing 3D solid models, more than two order-of-magnitude reduction in computational time has been demonstrated. The numerical results of small signal gas damping and spring effects have shown in good agreement with experimental results. Examples of large-amplitude transient analysis and comparison to experimental results are also provided.

\section{ACKNOWLEDGEMENTS}

The authors would like to thank Lucent Technology and Motorola SP for providing experimental data. The valuable discussion with Dr. Romanowicz and Dr. da Silva regarding to HDL and MemDamping is greatly appreciated.

\section{REFERENCES}

1. W. E. Langlois, "Isothermal Squeeze Films," Quar. Applied Mathematics, Vol. XX, No. 2, 1962, pp. 131-150.

2. J.J. Blech, "On Isothermal Squeeze Films", Journal of Lubrication Technology, Vol. 105, 1983, 615-620.

3. Y.-J. Yang and S. D. Senturia, "Numerical Simulation of Compressible Squeezed-Film Damping," Tech. Digest, Solid State Sensor and Actuator Workshop Hilton Head Island, SC, June 1996 ,

4. T. Veijola, et. al., "Model for Gas Filn! Damping in a Silicon Accelerometer", Proceedings Transducers 97 Vol. II, Chicago, IL, USA June 16-19, 1997, pp. 1097-1100

5. Y-J. Yang, et. al., "Effects of Air Damping on the Dynamics of Nonuniform Deformations of Microstructures", Transducers 97 Vol. II, Chicago, IL, USA June 16-19 1997, 1093-1096

6. T. Veijola, "Finite-Difference Large Displacement Gas-Film Model", Transducers 99, Sendai, Japan, Jun 7-10, 1999, pp11521155 .

7. M. da Silva, et. al., "Gas Damping and Sprng Effects on MEMS Devices with Multiple Perforations and Multiple Gaps", Transducers '99, Sendai, Japan, Jun 7-10, 1999, pp1148-1151.

8. M-A. Gretillat, "Electrostatic Polysilicon Micro-relays" Ph.D. Thesis, IMT, University of Neuchâtel, Switzerland 1997.

9. MEMCAD 4 Module Guide, Microcosm Technology Inc. 1999.

10. A. Odabasioglu, el. al., "PRIMA," IEEE Transaction on ComputerAided Dedign of Integrated Circuits and Systems, Vol. 17, No. 8 , August 1998.

11. M.H. Zaman, et. al. "A Technique for Extraction of Macro-Models in System level Simulation of Inertial Electro-Mechanical Micro Systems", MSM '99 pp 163-167. April 19-21 San Juan PR.

12. F. Wang and J. White, "Automatic Model Order Reduction fo a Microdevice using the Arnoldi Approach," ASME IMECE 98, DSC-Vol. 66, pp527-530.

13. G. K. Ananthasuresh, et. al., "An Approach to Macromodeling of MEMS for Nonlinear Dynamic Simulation," ASME International Mechanical Engineering Congress and Exposition, Symposium on MEMS, Atlanta, GA, Nov. 1996, pp. 18-22

14. K. Minami, et. al. "Simple Modeling and Simulation of the Squeeze Film Effect and Transient Response of The MEMS Device," MEMS 99, pp. 338-343.

15. D.S. Greywall, el. al., "Phenomenological Model for Gas Damping of Micromechanical Structures", Private Communication, Lucent Technologies, NJ 1998 